\title{
Molecular interpretation of mechanical behavior in four basic crystal packing of Isoniazid with homologous cocrystal formers
}

Jay Prakash Yadav, Ram Naresh Yadav, Piyush Uniyal, Hongbo Chen, Chenguang Wang, Changquan Calvin Sun, Navin Kumar, Arvind K. Bansal, Sanyog Jain

\section{Electronic Supplementary Information (ESI)}

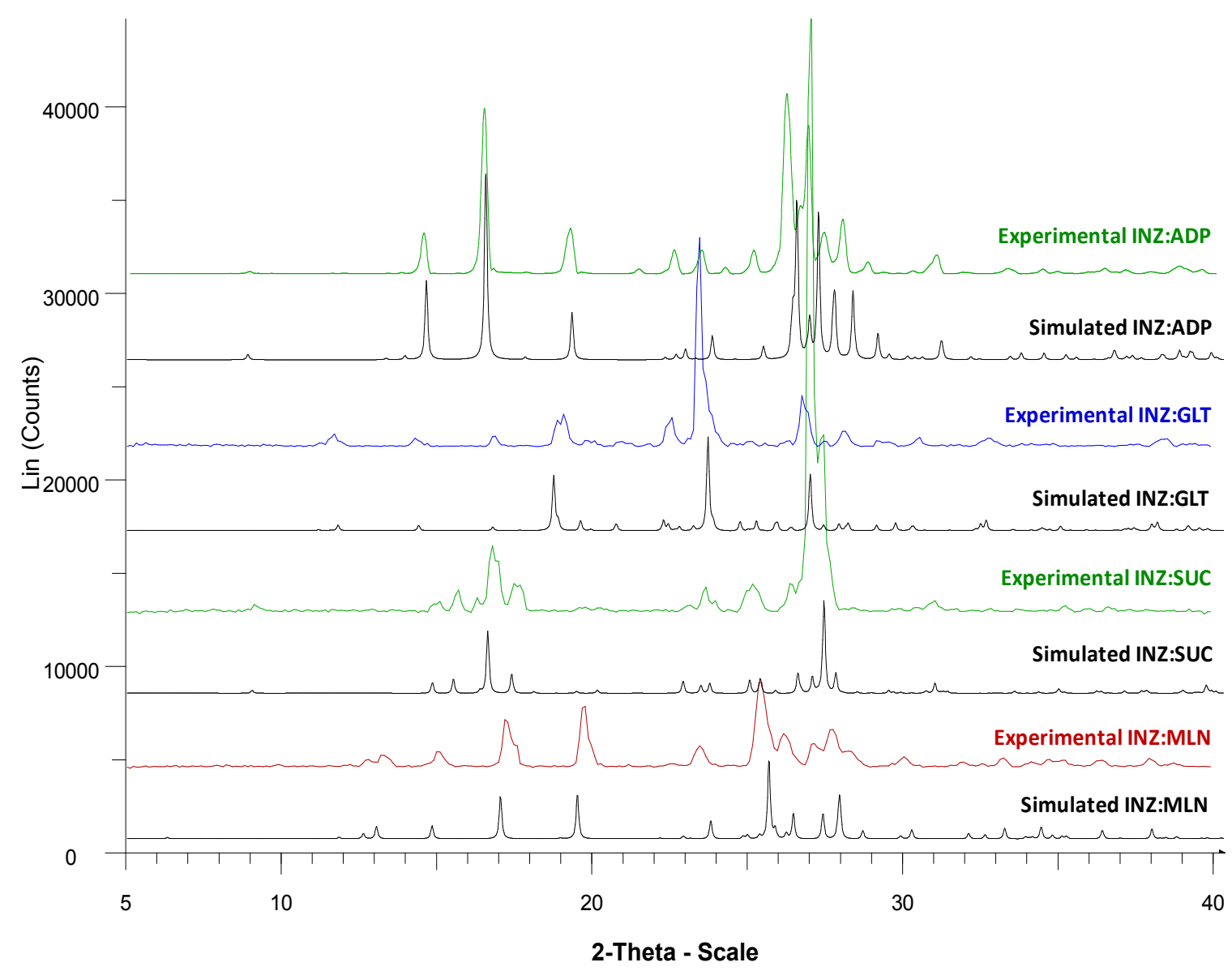

Figure S 1. Overlay of experimental and simulated (CSD ref-Code) powder X-ray diffraction (PXRD) patterns of generated cocrystals for mechanical behavior to establish their phase purity of solids to mechanical behavior. From bottom to top: INZ:MLN(2:1) (ref-Code: FADGEY), INZ:SUC(2:1) (ref-Code: FADGIC), INZ:GLT(1:1) (ref-Code: FADGOI), INZ:ADP(2:1) (ref-Code: FADGUO). 


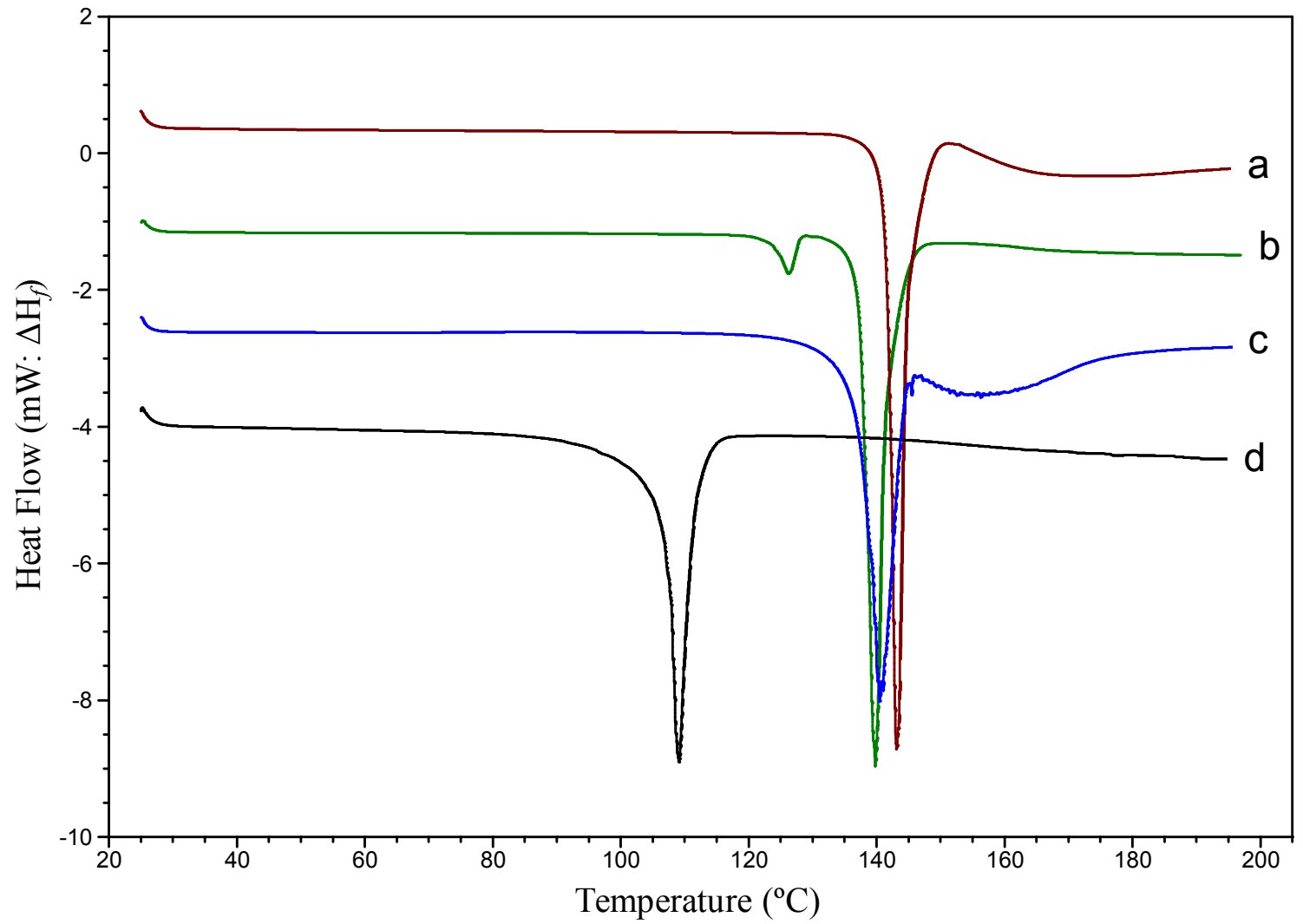

Figure S 2. Endothermic heat flow $(\mathrm{mW})$ on melting of Isoniazid (INZ) Cocrystals: (a) INZ:SUC; $\mathrm{T}_{\mathrm{m}}=143.09{ }^{\circ} \mathrm{C}$ $\Delta \mathrm{H}_{f}=203.60 \mathrm{~J} / \mathrm{g}$, (b) INZ:ADP; $\mathrm{T}_{\mathrm{m}}=139.78{ }^{\circ} \mathrm{C} \Delta \mathrm{H}_{f}=189.40 \mathrm{~J} / \mathrm{g}$, (c) INZ:MLN; $\mathrm{T}_{\mathrm{m}}=140.49{ }^{\circ} \mathrm{C} \Delta \mathrm{H}_{f}=125.70 \mathrm{~J} / \mathrm{g}$, and (d) INZ:GLT; $\mathrm{T}_{\mathrm{m}}=109.12^{\circ} \mathrm{C} \Delta \mathrm{H}_{f}=137.30 \mathrm{~J} / \mathrm{g}$

\section{Particle and bulk level characterization}

Table S 1. Particle and bulk level attributes of crystalline solids (std. dev. are shown in parenthesis)

\begin{tabular}{|c|c|c|c|c|c|}
\hline \multirow{2}{*}{ Solid } & \multirow{2}{*}{$\begin{array}{l}\text { True density } \\
\qquad(\mathrm{g} / \mathrm{cc})\end{array}$} & \multicolumn{2}{|c|}{$\begin{array}{l}\text { Particle size distribution } \\
\qquad(\mu \mathrm{m})\end{array}$} & \multirow{2}{*}{$\begin{array}{l}\text { Specific } \\
\text { surface area } \\
\left(\mathrm{m}^{2} / \mathrm{g}\right)\end{array}$} & \multirow{2}{*}{$\begin{array}{l}\text { Moisture } \\
\text { content (\% } \\
\text { w/w) }\end{array}$} \\
\hline & & $\mathrm{D}_{50}$ & $\mathrm{D}_{90}$ & & \\
\hline INZ:MLN & N $1.489( \pm 0.006)$ & 29.6 & 45.1 & $1.259( \pm 0.07)$ & 0.144 \\
\hline INZ:SUC & $1.459( \pm 0.004)$ & 27.2 & 48.3 & $1.626( \pm 0.03)$ & 0.120 \\
\hline INZ:GLT & $1.429( \pm 0.004)$ & 26.8 & 50.2 & $1.462( \pm 0.08)$ & 0.139 \\
\hline INZ:ADP & $1.427( \pm 0.005)$ & 25.7 & 42.9 & $1.721( \pm 0.05)$ & 0.109 \\
\hline
\end{tabular}

